\title{
Sparsity-Constrained Controllability Maximization With Application to Time-Varying Control Node Selection
}

\author{
$\operatorname{AUTHOR(S):~}$
}

Ikeda, Takuya; Kashima, Kenji

\section{CITATION:}

Ikeda, Takuya ...[et al]. Sparsity-Constrained Controllability Maximization With Application to Time-Varying Control Node Selection. IEEE Control Systems Letters 2018, 2(3): 321-326

\section{ISSUE DATE:}

2018-07

URL:

http://hdl.handle.net/2433/245128

\section{RIGHT:}

(C) 2018 IEEE. Personal use of this material is permitted. Permission from IEEE must be obtained for all other uses, in any current or future media, including reprinting/republishing this material for advertising or promotional purposes,

creating new collective works, for resale or redistribution to servers or lists, or reuse of any copyrighted component of this work in other works.; This is not the published version. Please cite only the published version.; この論文は出版社版

でありません。引用の際には出版社版をご確認ご利用ください。 


\title{
Sparsity-constrained controllability maximization with application to time-varying control node selection
}

\author{
Takuya Ikeda, Kenji Kashima, Member, IEEE,
}

\begin{abstract}
In this paper, we consider the maximization of a quantitative metric of controllability with a constraint of $L^{0}$ norm of the control input. Since the optimization problem contains a combinatorial structure, we introduce a convex relaxation problem for the sake of reducing computation burden. We prove the existence of solutions to the main problem and also give a simple condition under which the relaxed problem gives a solution to the main problem. It should be emphasized that the main problem can formulate time-varying control node selection, which attempts to extract when and where exogenous inputs should be provided in order to achieve high controllability of multi-agent systems.
\end{abstract}

Index Terms-Optimal control, Linear systems, Time-varying systems, Sparse optimal control, Networked control systems.

\section{INTRODUCTION}

$\mathbf{O}$ PTIMAL control problems in which control variables are penalized via the $L^{0}$-norm have attracted a renewed interest due to its connection to sparsity. Since the penalty cost is defined as the length of the support of controls, the optimization tends to make the control input identically zero on a set with positive measures, and the optimal control is switched off completely on parts of the time domain. This is why the problem is referred to as sparse optimal control. This optimal control framework is, for example, applied to actuator placements [1], [2], [3], multi-period investments [4], and networked control systems in the presence of packetdropouts [5], to name a few.

The sparse optimal control satisfying a given state transition from a point to another point is theoretically analyzed in [6], [7], [8]. On the other hand, it is also important to investigate a time duration over which some control inputs can realize efficient state transitions towards all directions. This enables us to find controller activation schedule that does not depend on the target state. In addition to input sparsity, one might be concerned with a question of how much energy is required to steer the system. For this purpose, several notions of controllability have been proposed as a measure of possibility of state transitions.

In view of this, in this paper, we investigate a novel optimal control problem that aims to maximize a quantitative metric of controllability when control inputs are constrained in terms of the $L^{0}$ norm. For the metric, this paper adopts the trace of the controllability Gramian from the analytical perspective. This

T. Ikeda and K. Kashima are with Graduate School of Informatics, Kyoto University, Kyoto, 606-8501, Japan. (e-mail: ikeda.t@bode.amp.i.kyotou.ac.jp, kk@i.kyoto-u.ac.jp) quantity is closely related to the average energy required to steer the system in all directions in the state space. Here, it is worthy of note that the metric is widely used in the context of node selection problem [9], [10], [11], [12]. In the context, our optimal solution is interpreted as an answer to the question of when and where exogenous control inputs should be provided for high controllability. Hence the present paper corresponds to the case of time-varying node selection; see Section V. We also note that this paper penalizes both the $L^{0}$ cost and the $L^{2}$ cost with respect to the control signal, and this approach is related to sparse quadratic regulator treated in [13].

The problem addressed above, however, includes a combinatorial structure. To circumvent this, we introduce a convex optimization problem for the sake of reduction of computation burden. For the theoretical analysis, we first reformulate the convex optimization so that Pontryagin's minimum principle is applicable, and then characterize the optimal solution by using the costate. Based on this characterization, we establish a condition for the main problem to be exactly solved via the convex optimization. Thanks to the result, the existence of optimal solutions to the main problem is also shown.

The remainder of this paper is organized as follows: In Section II, we give mathematical preliminaries for our subsequent discussion. In section III, we formulate our optimal control problem. Section IV gives a theoretical analysis. We show a condition for the problem to boil down to a convex optimization problem and prove the existence of optimal solutions to the main problem. Section V illustrates the application of the main problem to node selection problem, and we offer concluding remarks in Section VI.

\section{Mathematical Preliminaries}

This section reviews notation that will be used throughout the paper.

Let $m$ be a positive integer and $\Omega$ be a subset of $\mathbb{R}$. For a vector $a=\left[a_{1}, a_{2}, \ldots, a_{m}\right]^{\top} \in \mathbb{R}^{m}, \operatorname{diag}(a)$ denotes the diagonal matrix whose $(i, i)$-component is given by $a_{i}$, and $a \in \Omega^{m}$ means $a_{i} \in \Omega$ for all $i$. We denote the Euclidean norm by $\|a\| \triangleq\left(\sum_{i=1}^{m} a_{i}^{2}\right)^{1 / 2}$. Let $N_{1}$ and $N_{2}$ be positive integers. For a matrix $M \in \mathbb{R}^{N_{1} \times N_{2}}, M^{\top}$ denotes the transpose of $M$, and $\operatorname{Tr} M$ denotes the trace of $M$.

Let $T>0$. We define the $L^{0}$ norm and $L^{1}$ norm of a continuous-time signal $v(t)=\left[v_{1}(t), v_{2}(t), \ldots, v_{m}(t)\right]^{\top} \in$ 
$\mathbb{R}^{m}$ over a time interval $[0, T]$ by

$$
\begin{aligned}
& \|v\|_{0} \triangleq \sum_{j=1}^{m} \mu_{L}\left(\left\{t \in[0, T]: v_{j}(t) \neq 0\right\}\right), \\
& \|v\|_{1} \triangleq \sum_{j=1}^{m} \int_{0}^{T}\left|v_{j}(t)\right| d t
\end{aligned}
$$

where $\mu_{L}$ is the Lebesgue measure on $\mathbb{R}$. We denote the set of all $v$ with $\|v\|_{1}<\infty$ by $L^{1}$.

\section{Problem Formulation}

\section{A. Controllability Metrics}

This paper investigates an optimal control problem that maximizes a metric of controllability with a sparse control. Let us first define a linear model as follows:

$$
\dot{x}(t)=A x(t)+B u(t), \quad 0 \leq t \leq T,
$$

where $x(t) \in \mathbb{R}^{n}$ is the state; $u(t) \in \mathbb{R}^{m}$ is the control input; $A \in \mathbb{R}^{n \times n}$ and $B \in \mathbb{R}^{n \times m}$ are constant matrices; and $T>0$ is the final time of control. This paper is interested in a sparse control constrained in terms of the $L^{0}$ norm by $\|u\|_{0} \leq \alpha$ with a given positive number $\alpha>0$. This results in the following expression of the system (1):

$$
\begin{aligned}
& \dot{x}(t)=A x(t)+B V(t) u(t), \quad 0 \leq t \leq T, \\
& V(t) \triangleq \operatorname{diag}(v(t)),
\end{aligned}
$$

where $v(t) \triangleq\left[v_{1}(t), v_{2}(t), \ldots, v_{m}(t)\right]^{\top} \in \mathbb{R}^{m}$ is a timevarying vector satisfying $\|v\|_{0} \leq \alpha$ and $v(t) \in\{0,1\}^{m}$ for all $t \in[0, T]$. Note that the function $v(t)$ represents the activation schedule of the control input. More precisely, the $j$-th variable of the control input is able to affect the system at time $t$ if and only if $v_{j}(t)=1$.

In what follows, we introduce a metric of the controllability for the system (2). The classical notion of controllability denotes whether the system can be driven to any desired state from any initial state by using an appropriate control input. More precisely, the system (2) is said to be controllable on $[0, T]$ if for any $x_{0}, x_{f} \in \mathbb{R}^{n}$ there exists a control input $u(t)$ such that the state of the system $x(t)$ is driven from $x(0)=x_{0}$ to $x(T)=x_{f}$ by using the control input $u(t)$. It is well known that the system is controllable if and only if the following matrix called controllability Gramian is non-singular:

$$
W_{c} \triangleq \int_{0}^{T} e^{A t} \mathcal{B}(t) \mathcal{B}(t)^{\top} e^{A^{\top} t} d t
$$

where $\mathcal{B}(t) \triangleq B V(t) \in \mathbb{R}^{n \times m}$. Note that this classical controllability is a binary measure that determines whether the system is controllable or not, and it does not evaluate how easy the system is to control. Even if the system is theoretically controllable, control inputs might require high energy cost, which fails to realize desired state transitions in practice. Then, controllability measures that quantify the required energy cost of steering the system have been analyzed. We here recall the minimum-energy control problem:

$$
\begin{array}{cl}
\underset{u}{\operatorname{minimize}} & \int_{0}^{T}\|u(t)\|^{2} d t \\
\text { subject to } & \dot{x}(t)=A x(t)+\mathcal{B}(t) u(t), \\
& x(0)=x_{0}, \quad x(T)=0 .
\end{array}
$$

The minimum control energy is then given by $x_{0}^{\top} W_{c}^{-1} x_{0}$ [14]. Based on this, recent works have been considered to make $W_{c}$ as "large" as possible, and a number of controllability metrics have been proposed, including $\operatorname{Tr} W_{c}, \lambda_{\min }\left(W_{c}\right), \operatorname{det}\left(W_{c}\right)$, and $\operatorname{rank}\left(W_{c}\right)$; see e.g. [9], [10]. While each measure has its own advantage, this paper adopts the trace of the controllability Gramian $\operatorname{Tr} W_{c}$ from the analytical perspective. This quantity is closely related to the average controllability on the ball $\left\{x_{0}\right.$ : $\left.\left\|x_{0}\right\|=1\right\}$ by the following equation [15]:

$$
\frac{\int_{\left\|x_{0}\right\|=1} x_{0}^{\top} W_{c}^{-1} x_{0} d x_{0}}{\int_{\left\|x_{0}\right\|=1} d x_{0}}=\frac{1}{n} \operatorname{Tr} W_{c}^{-1} .
$$

\section{B. Main Problem}

In this paper, we consider one problem: how should we provide control inputs to the system in order to realize the best controllability when control inputs are constrained in terms of the $L^{0}$ norm? The problem is formulated as the following optimization:

Problem 1: Given $A \in \mathbb{R}^{n \times n}, B \in \mathbb{R}^{n \times m}, T>0$, and $\alpha>0$, find a time-varying matrix $V(t) \triangleq \operatorname{diag}(v(t)), v(t) \triangleq$ $\left[v_{1}(t), v_{2}(t), \ldots, v_{m}(t)\right]^{\top}$, that solves

$$
\begin{array}{ll}
\underset{v_{1}, v_{2}, \ldots, v_{m}}{\operatorname{maximize}} & J(V) \triangleq \operatorname{Tr} \int_{0}^{T} e^{A t} B V(t) V(t)^{\top} B^{\top} e^{A^{\top} t} d t \\
\text { subject to } & v(t) \in\{0,1\}^{m} \quad \forall t \in[0, T], \quad\|v\|_{0} \leq \alpha .
\end{array}
$$

We are now interested in the average controllability as mentioned in subsection III-A. Hence the cost function in the optimization problem is defined by the trace of the controllability Gramian according to the system (2).

Let us now reformulate Problem 1 for the subsequent theoretical analysis.

Lemma 1: Problem 1 is equivalent to the following problem:

$$
\begin{array}{ll}
\underset{v_{1}, v_{2}, \ldots, v_{m}}{\operatorname{maximize}} & J_{1}(V) \\
\text { subject to } & v(t) \in\{0,1\}^{m} \quad \forall t \in[0, T], \quad\|v\|_{0} \leq \alpha .
\end{array}
$$

where

$$
\begin{aligned}
& J_{1}(V) \triangleq \int_{0}^{T}\left[f_{1}(t), f_{2}(t), \ldots, f_{m}(t)\right] v(t) d t, \\
& f_{j}(t) \triangleq b_{j}^{\top} e^{A^{\top} t} e^{A t} b_{j}, \quad j=1,2, \ldots, m, \\
& v(t) \triangleq\left[v_{1}(t), v_{2}(t), \ldots, v_{m}(t)\right]^{\top},
\end{aligned}
$$

and $b_{j}$ is the $j$-th column of $B$. 
Proof: It is enough to show $J(V)=J_{1}(V)$ for any $v$ that satisfies the constraints. It follows from a property of the trace operator that

$$
\begin{aligned}
J(V) & =\operatorname{Tr} \int_{0}^{T} e^{A t} B V(t) V(t)^{\top} B^{\top} e^{A^{\top} t} d t \\
& =\operatorname{Tr} \int_{0}^{T} B^{\top} e^{A^{\top} t} e^{A t} B V(t)^{2} d t
\end{aligned}
$$

Here, we have $V(t)^{2}=V(t)$, since a constraint in Problem 1 imposes the variables $v_{i}$ 's to take only the values of 0 and 1 . We thus obtain the equivalent form (3) of Problem 1.

In this paper, we show that optimal solutions to Problem 1 can be obtained by solving a convex optimal control problem.

\section{ANALYSIS}

We first introduce a convex relaxed problem of Problem 1.

Problem 2: Given $A \in \mathbb{R}^{n \times n}, B \in \mathbb{R}^{n \times m}, T>0$, and $\alpha>0$, find a time-varying matrix $V(t) \triangleq \operatorname{diag}(v(t)), v(t) \triangleq$ $\left[v_{1}(t), v_{2}(t), \ldots, v_{m}(t)\right]^{\top}$, that solves

$$
\begin{array}{ll}
\underset{v_{1}, v_{2}, \ldots, v_{m}}{\operatorname{maximize}} & J_{1}(V) \\
\text { subject to } & v(t) \in[0,1]^{m} \quad \forall t \in[0, T], \quad\|v\|_{1} \leq \alpha .
\end{array}
$$

We first show the discreteness of solutions to Problem 2, which guarantees that the optimal solutions to Problem 2 satisfy the constraints in Problem 1, which will be illustrated in the proof of Theorem 2. For this purpose, we recall Pontryagin's maximum principle [16, Theorem 22.2].

Proposition 1: Consider the following optimal control problem:

$$
\begin{array}{cl}
\underset{w}{\operatorname{minimize}} & \int_{0}^{T} \ell(t, w(t)) d t \\
\text { subject to } & \dot{z}(t)=F z(t)+G w(t), t \in[0, T] \text { a.e. } \\
& w(t) \in[0,1]^{m}, t \in[0, T] \text { a.e. } \\
& z(0)=z_{0}, z(T) \in E,
\end{array}
$$

where $\ell$ is continuous, $z(t) \in \mathbb{R}, w(t) \in \mathbb{R}^{m}, F \in \mathbb{R}$, $G \in \mathbb{R}^{1 \times m}, T>0, z_{0} \in \mathbb{R}$, and $E \subset \mathbb{R}$. Note that $\left(\ell, F, G, T, z_{0}, E\right)$ is given. Define the Hamiltonian function $H^{\eta}:[0, T] \times \mathbb{R} \times \mathbb{R} \times \mathbb{R}^{m}$ associated to Problem (OC) by

$$
H^{\eta}(t, z, p, w) \triangleq p(F z+G w)-\eta \ell(t, w)
$$

where $\eta$ is either 0 or 1 . Let the process $\left(z_{*}, w_{*}\right)$ be a local minimizer for Problem (OC). Then, there exists an arc $p$ : $[0, T] \rightarrow \mathbb{R}$ and a scalar $\eta$ equal to 0 or 1 satisfying the nontriviality condition:

$$
(\eta, p(t)) \neq 0 \quad \forall t \in[0, T],
$$

the adjoint equation for almost every $t$ :

$$
-\dot{p}(t)=\frac{\partial H^{\eta}}{\partial z}\left(t, z_{*}(t), p(t), w_{*}(t)\right),
$$

as well as the maximum condition for almost every $t$ :

$$
H^{\eta}\left(t, z_{*}(t), p(t), w_{*}(t)\right)=\sup _{w \in[0,1]^{m}} H^{\eta}\left(t, z_{*}(t), p(t), w\right) .
$$

From now on, we show the discreteness of optimal solutions to Problem 2.

Theorem 1 (discreteness): Assume that $f_{j}(t)$ defined in Lemma 1 is not constant on $[0, T]$ for all $j \in\{1,2, \ldots, m\}$. Then any solution to Problem 2 takes only 0 and 1 almost everywhere.

Proof: We first reformulate Problem 2 into a form to which Pontryagin's maximum principle is applicable. For any $v$ such that $v(t) \in[0,1]^{m}$ on $[0, T]$, we have

$$
\begin{aligned}
\|v\|_{1} & =\sum_{j=1}^{m} \int_{0}^{T}\left|v_{j}(t)\right| d t=\sum_{j=1}^{m} \int_{0}^{T} v_{j}(t) d t \\
& =\int_{0}^{T}[1,1, \ldots, 1] v(t) d t,
\end{aligned}
$$

where $v(t)=\left[v_{1}(t), v_{2}(t), \ldots, v_{m}(t)\right]^{\top}$. Note that the value is equal to the final state $y(T)$ of the system

$$
\dot{y}(t)=[1,1, \ldots, 1] v(t)
$$

with $y(0)=0$. Hence the set of all variables satisfying the constraints in Problem 2 is equivalent to the set

$$
\begin{aligned}
\{v: v(t) & \in[0,1]^{m} \quad \forall t \in[0, T], \\
\dot{y}(t) & =[1,1, \ldots, 1] v(t), \quad y(0)=0, \quad y(T) \leq \alpha\},
\end{aligned}
$$

and Problem 2 is equivalently expressed by the following problem:

$$
\begin{array}{ll}
\underset{v_{1}, v_{2}, \ldots, v_{m}}{\operatorname{maximize}} & J_{1}(V) \\
\text { subject to } & \dot{y}(t)=[1,1, \ldots, 1] v(t), \\
& y(0)=0, \quad y(T) \leq \alpha, \\
& v(t) \in[0,1]^{m} \quad \forall t \in[0, T] .
\end{array}
$$

We thus obtain an optimal control problem to which Pontryagin's maximum principle is applicable.

Define the Hamiltonian function $H^{\eta}:[0, T] \times \mathbb{R} \times \mathbb{R} \times \mathbb{R}^{m}$ associated to the problem (7) by

$$
H^{\eta}(t, y, p, v) \triangleq p[1,1, \ldots, 1] v-\eta \ell(t, v),
$$

where $\eta$ is either 0 or 1 and

$$
\begin{aligned}
& \ell(t, v) \triangleq-\left[f_{1}(t), f_{2}(t), \ldots, f_{m}(t)\right] v, \\
& f_{j}(t) \triangleq b_{j}^{\top} e^{A^{\top} t} e^{A t} b_{j}, \quad j=1,2, \ldots, m .
\end{aligned}
$$

Let the process $\left(y_{*}, v_{*}\right)$ be a local maximizer for the problem (7). Then there exists an arc $p:[0, T] \rightarrow \mathbb{R}$ and a scalar $\eta$ equal to 0 or 1 satisfying the conditions (4), (5), and (6). Put the set on which (4), (5), and (6) hold by $I \subset[0, T]$. Note that $\mu_{L}(I)=T$. Since we now have $\frac{\partial H^{\eta}}{\partial y}=0$, it follows from (5) that there exists $p_{0} \in \mathbb{R}$ and $p(t)=p_{0}$ for $t \in I$. Then, it follows from (6) that

$$
\left(p_{0}+\eta f_{j}(t)\right) v_{j}^{*}(t)=\sup _{v_{j} \in[0,1]}\left(p_{0}+\eta f_{j}(t)\right) v_{j}
$$

for each $j \in\{1,2, \ldots, m\}$ and $t \in I$. Note that the supremum is attained by a point in $[0,1]$, since the right hand side is a linear function of $v_{j}$ on a closed interval. Hence

$$
v_{j}^{*}(t)=\underset{v_{j} \in[0,1]}{\arg \max }\left(p_{0}+\eta f_{j}(t)\right) v_{j} .
$$


The characterization is divided into the following two cases.

1) If $\eta=0$, then $p_{0} \neq 0$ from (4). Hence

$$
v_{j}^{*}(t)=\underset{v_{j} \in[0,1]}{\arg \max } p_{0} v_{j}= \begin{cases}1, & \text { if } p_{0}>0, \\ 0, & \text { if } p_{0}<0 .\end{cases}
$$

2) If $\eta=1$, then

$$
v_{j}^{*}(t)=\underset{v_{j} \in[0,1]}{\arg \max }\left(p_{0}+f_{j}(t)\right) v_{j} .
$$

Hence

$$
v_{j}^{*}(t)= \begin{cases}1, & \text { if } p_{0}+f_{j}(t)>0 \\ 0, & \text { if } p_{0}+f_{j}(t)<0\end{cases}
$$

and $v_{j}^{*}(t)$ is not determined if $p_{0}+f_{j}(t)=0$. Here, put $I_{j} \triangleq\left\{t \in[0, T] \cap I: p_{0}+f_{j}(t)=0\right\}$.

In what follows, we show that for each $j$, if $f_{j}(t)$ is not constant, then $v_{j}^{*}$ takes only 0 and 1 almost everywhere. For the purpose, we show that if $\eta=1$ and $\mu_{L}\left(I_{j_{0}}\right)>0$ for some $j_{0}$, then $f_{j_{0}}(t)$ is constant. Put

$$
\phi_{j_{0}}(t) \triangleq p_{0}+f_{j_{0}}(t) \text {. }
$$

Then, $\phi_{j_{0}}(t)=0$ for $t \in I_{j_{0}}$. Since $\phi_{j_{0}}(t)$ is an analytic function, it follows from $\mu_{L}\left(I_{j_{0}}\right)>0$ that $\phi_{j_{0}} \equiv 0$, as described in [17]. This implies that $f_{j_{0}}(t)$ is constant, and then completes the proof.

Remark 1: As illustrated in the proof, the $j$-th component $v_{j}^{*}(t)$ of the optimal solution may switch the values only at time $t$ such that $f_{j}(t)+p_{0}=0$. Since the function $f_{j}(t)+p_{0}$ is analytic, the set of all zeros of the function does not contain accumulation points, which ensures the nonexistence of Zeno phenomena.

The following theorem is the main result, which shows the equivalence between Problem 1 and Problem 2.

Theorem 2 (equivalence): Assume that $f_{j}(t)$ defined in Lemma 1 is not constant for all $j$. Denote the set of all solutions to Problem 1 and Problem 2 by $\mathcal{V}_{1}^{*}$ and $\mathcal{V}_{2}^{*}$, respectively. If $\mathcal{V}_{2}^{*} \neq \emptyset$, then $\mathcal{V}_{1}^{*}=\mathcal{V}_{2}^{*}$.

Proof: Let $\hat{v} \triangleq\left[\hat{v}_{1}, \hat{v}_{2}, \ldots, \hat{v}_{m}\right]^{\top}$ be an optimal solution to Problem 2. Then all $\hat{v}_{j}$ 's take only the values of 0 and 1 almost everywhere by Theorem 1 . Note that the null set $\cup_{j=1}^{m}\left\{t \in[0, T]: \hat{v}_{j}(t) \notin\{0,1\}\right\}$ does not affect the cost, and hence we can adjust the variables so that $\hat{v}_{j}(t) \in\{0,1\}$ on $[0, T]$ for all $j$, without loss of the optimality. We have

$$
\begin{aligned}
\sum_{j=1}^{m}\left\|\hat{v}_{j}\right\|_{1} & =\sum_{j=1}^{m} \int_{\left\{t \in[0, T]: \hat{v}_{j}(t) \neq 0\right\}}\left|\hat{v}_{j}(t)\right| d t \\
& =\sum_{j=1}^{m}\left\|\hat{v}_{j}\right\|_{0},
\end{aligned}
$$

where we used the discreteness of $\hat{v}_{j}$. Since $\hat{v}$ satisfies the constraints in Problem 2, we have $\sum_{j=1}^{m}\left\|\hat{v}_{j}\right\|_{0} \leq \alpha$ from (9). Thus, $\hat{v}$ also satisfies the constraints in Problem 1.

Here,

$$
\max _{\substack{v(t) \in\{0,1\}^{m} \\\|v\|_{0} \leq \alpha}} J_{1}(V) \leq \max _{\substack{v(t) \in[0,1]^{m} \\\|v\|_{0} \leq \alpha}} J_{\forall t} J_{1}(V) .
$$

Since we have $\|g\|_{1} \leq\|g\|_{0}$ for any measurable function $g(t) \in \mathbb{R}$ with $g(t) \in[0,1]$ on $[0, T]$, we have

$$
\begin{aligned}
& \left\{v: v(t) \in[0,1]^{m} \forall t,\|v\|_{0} \leq \alpha\right\} \\
& \subset\left\{v: v(t) \in[0,1]^{m} \forall t,\|v\|_{1} \leq \alpha\right\} .
\end{aligned}
$$

Hence

$$
\max _{\substack{v(t) \in[0,1]^{m} \\\|v\|_{0} \leq \alpha}} J_{1}(V) \leq \max _{\substack{v(t) \in[0,1]^{m} \\\|v\|_{1} \leq \alpha}} J_{i}(V) .
$$

It follows from inequalities (10) and (11) that

$$
\max _{\substack{v(t) \in\{0,1\}^{m} \\\|v\|_{0} \leq \alpha}} J_{1}(V) \leq \max _{\substack{v(t) \in[0,1]^{m} \\\|v\|_{1} \leq \alpha}} J_{\forall t} J_{1}(V) .
$$

Here, $\hat{v}$ is an optimal solution to Problem 2, and hence

$$
\max _{\substack{v(t) \in[0,1]^{m} \\\|v\|_{1} \leq \alpha}} J_{1}(V)=J_{1}\left(V_{2}^{*}\right)
$$

where $V_{2}^{*} \triangleq \operatorname{diag}(\hat{v})$. On the other hand, since $\hat{v}$ satisfies the constraints in Problem 1, we have

$$
J_{1}\left(V_{2}^{*}\right) \leq \max _{\substack{v(t) \in\{0,1\}^{m} \\\|v\|_{0} \leq \alpha}} J_{1}(V) .
$$

Hence, we have

$$
J_{1}\left(V_{2}^{*}\right)=\max _{\substack{v(t) \in\{0,1\}^{m} \\\|v\|_{0} \leq \alpha}} J_{1}(V)
$$

by (12), (13), and (14). This means that $\hat{v}$ is a solution to Problem 1. Hence we have $\mathcal{V}_{2}^{*} \subset \mathcal{V}_{1}^{*}$ and $\mathcal{V}_{1}^{*} \neq \emptyset$.

Let us take any optimal solution $\tilde{v} \triangleq\left[\tilde{v}_{1}, \tilde{v}_{2}, \ldots, \tilde{v}_{m}\right]^{\top} \in$ $\mathcal{V}_{1}^{*}$. Then we have $\tilde{v}_{j}(t) \in\{0,1\}$ on $[0, T]$ for all $j$ and

$$
\sum_{j=1}^{m}\left\|\tilde{v}_{j}\right\|_{1}=\sum_{j=1}^{m}\left\|\tilde{v}_{j}\right\|_{0} \leq \alpha .
$$

Hence $\tilde{v}$ also satisfies the constraints in Problem 2. In addition, it follows from (15) that $J_{1}\left(V_{1}^{*}\right)=J_{1}\left(V_{2}^{*}\right)$, where $V_{1}^{*} \triangleq$ $\operatorname{diag}(\tilde{v})$. Therefore, $\tilde{v} \in \mathcal{V}_{2}^{*}$ and $\mathcal{V}_{1}^{*} \subset \mathcal{V}_{2}^{*}$. This gives $\mathcal{V}_{1}^{*}=\mathcal{V}_{2}^{*}$.

Remark 2: When a function $f_{j}$ is constant for some $j$, optimal solutions to Problem 2 do not necessarily take only values of 0 and 1 . Indeed, once the $j$-th component of an optimal solution to Problem 2 takes the value 1 on an interval, there exists an optimal solution whose $j$-th component takes besides the values of 0 and 1 . This implies that the set $\mathcal{V}_{2}^{*}$ is not necessarily included in the set $\mathcal{V}_{1}^{*}$ when a function $f_{j}$ is constant.

In Theorem 2, we assume the existence of optimal solutions to Problem 2 in order to discuss the equivalence $\mathcal{V}_{1}^{*}=\mathcal{V}_{2}^{*}$. We next show the existence of solutions to Problem 1 and Problem 2. For the purpose, we first show that there exist solutions to Problem 2. Note that this guarantees the existence of solutions to Problem 1 if $f_{j}(t)$ is not constant for all $j$ by Theorem 2. We then finally show that there exists at least one solution to Problem 1 , even if $f_{j}(t)$ is constant for some $j$.

Theorem 3 (existence): For any $A \in \mathbb{R}^{n \times n}, B \in \mathbb{R}^{n \times m}$, $T>0$, and $\alpha>0$, there exists at least one optimal solution to Problem 1 and Problem 2, respectively. 
Proof: We first show the existence of solutions to Problem 2. For this, we consider the equivalent problem (7). Let us denote the set of all variables satisfying the constraints in the problem (7) by $\mathcal{V}$, i.e,

$$
\begin{array}{r}
\mathcal{V} \triangleq\left\{v \in L^{1}: \int_{0}^{T}[1,1, \ldots, 1] v(t) d t \leq \alpha,\right. \\
\left.v(t) \in[0,1]^{m} \forall t \in[0, T]\right\} .
\end{array}
$$

Since $\mathcal{V} \neq \emptyset$, we can define

$$
\theta \triangleq \sup \left\{J_{1}(V): v \in \mathcal{V}\right\}
$$

Then there exists a sequence $\left\{v^{(l)}\right\}_{l \in \mathbb{N}} \subset \mathcal{V}$ such that $\lim _{l \rightarrow \infty} J_{1}\left(v^{(l)}\right)=\theta$. Here, the set $\left\{w \in L^{\infty}:\|w\|_{\infty} \leq 1\right\}$ is sequentially compact in the weak ${ }^{*}$ topology of $L^{\infty}$ [18]. Then it follows that there exist a measurable function $v^{(\infty)}$ with $v^{(\infty)}(t) \in[0,1]^{m}$ almost everywhere and a subsequence $\left\{v^{\left(l^{\prime}\right)}\right\}$ such that each component $v_{j}^{\left(l^{\prime}\right)}$ converges to $v_{j}^{(\infty)}$, the $j$-th component of $v^{(\infty)}$, in the weak ${ }^{*}$ topology of $L^{\infty}$, that is, we have

$$
\lim _{l^{\prime} \rightarrow \infty} \int_{0}^{T}\left(v_{j}^{\left(l^{\prime}\right)}(t)-v_{j}^{(\infty)}(t)\right) g(t) d t=0
$$

for any $g \in L^{1}$ and $j=1,2, \ldots, m$. Then

$$
\begin{aligned}
\int_{0}^{T}[1,1, \ldots, 1] v^{(\infty)}(t) d t & =\lim _{l^{\prime} \rightarrow \infty} \int_{0}^{T}[1,1, \ldots, 1] v^{\left(l^{\prime}\right)}(t) d t \\
& \leq \alpha
\end{aligned}
$$

and hence $v^{(\infty)} \in \mathcal{V}$. In addition, we have

$$
\begin{aligned}
J_{1}\left(V^{(\infty)}\right) & =\int_{0}^{T}\left[f_{1}(t), f_{2}(t), \ldots, f_{m}(t)\right] v^{(\infty)}(t) d t \\
& =\lim _{l^{\prime} \rightarrow \infty} \int_{0}^{T}\left[f_{1}(t), f_{2}(t), \ldots, f_{m}(t)\right] v^{\left(l^{\prime}\right)}(t) d t \\
& =\lim _{l^{\prime} \rightarrow \infty} J_{1}\left(V^{\left(l^{\prime}\right)}\right)=\theta
\end{aligned}
$$

where $V^{(\infty)} \triangleq \operatorname{diag}\left(v^{(\infty)}\right)$ and $V^{\left(l^{\prime}\right)} \triangleq \operatorname{diag}\left(v^{\left(l^{\prime}\right)}\right)$. This shows that $v^{(\infty)}$ is an optimal solution to Problem 2 .

We next show that there exists at least one optimal solution to Problem 2 that takes only the values of 0 and 1 even if $f_{j}(t)$ is constant for some $j$. Here, let us denote the set of all solutions to Problem 2 by $\mathcal{V}_{2}^{*}$, which is not empty from the discussion above, and take any optimal solution $v^{*} \in \mathcal{V}_{2}^{*}$. Now, it is enough to consider the case that $\eta=1$ and $K \triangleq$ $\left\{j \in\{1,2, \ldots, m\}: \mu\left(I_{j}\right)>0\right\} \neq \emptyset$, where $\eta$ and $I_{j}$ are defined in the proof of Theorem 1, since for the other cases $v^{*}$ obviously take only the values of 0 and 1 . Note that the $j$-th component $v_{j}^{*}$ of $v^{*}$ satisfies $v_{j}^{*}(t) \in\{0,1\}$ on $[0, T]$ for $j \notin K$. For any $j \in K$, it follows from the proof of Theorem 2 that $\phi_{j}(t) \equiv 0$, where $\phi_{j}$ is defined by (8). Hence $f_{j}(t)=-p_{0}$ on $[0, T]$ for all $j \in K$. Here we take a measurable function $v$ such that

$$
\begin{gathered}
\int_{0}^{T} v_{j}(t) d t=\int_{0}^{T} v_{j}^{*}(t) d t \\
v_{j}(t) \in\{0,1\} \quad \forall t \in[0, T]
\end{gathered}
$$

for $j \in K$ and $v_{j}=v_{j}^{*}$ for $j \notin K$. Then the function $v \triangleq$ $\left[v_{1}, v_{2}, \ldots, v_{m}\right]^{\top}$ satisfies $J_{1}(V)=J_{1}\left(V^{*}\right),\|v\|_{1}=\left\|v^{*}\right\|_{1} \leq$ $\alpha$, and $v(t) \in\{0,1\}^{m}$ on $[0, T]$, where $V=\operatorname{diag}(v)$ and $V^{*}=\operatorname{diag}\left(v^{*}\right)$. Hence $v$ is a solution to Problem 2 that takes only 0 and 1 .

Finally, the set of all solutions to Problem 2 that take only 0 and 1 is equal to $\mathcal{V}_{1}^{*}$, which is the set of all solutions to Problem 1. This can be verified by the proof of Theorem 2 . Thus, we obtain $\mathcal{V}_{1}^{*} \neq \emptyset$.

\section{Application to Control Node Selection}

In this section, we illustrate the application of Problem 1 to control node selection. We first briefly review the node selection problem. The purpose of the problem is to identify the set of nodes with exogenous control inputs that can effectively guide the system's entire dynamics. The selected nodes are called control nodes, and the selection techniques of control nodes have been extensively studied in the context of complex networks. In recent works, control nodes are chosen in order to optimize a metric of controllability [19], [20], [9], [10], [21], [22]. For example, [19] considers the minimum set of control nodes that guarantees the classical controllability proposed in [23]; [20] considers the structural controllability; and [9], [10] introduce quantities that evaluate how much the system is easy to control, such as the trace, the minimum eigenvalue, and the rank of the controllability Gramian. While these works investigate the selection problem in which the set of control nodes is fixed over the time, more recent works [11], [12] alternatively select the time-varying set of control nodes for discrete-time systems.

In the context of the selection problem, our model (2) is interpreted as follows: $x(t) \triangleq\left[x_{1}(t), x_{2}(t), \ldots, x_{n}(t)\right]^{\top}$ is the state vector of the network consisting of $n$ nodes, where $x_{i}(t)$ is the state of the $i$-th node at time $t ; u(t)$ is the exogenous control input that influences the network dynamics; $A$ is the dynamics matrix that represents the information flow among nodes. In particular, the node selection problem is interested in the design of the $B$-matrix, since it locates control nodes. In our case, $B V(t)$ plays the role. More precisely, if $v_{j}(t)=1$, then the input $u_{j}(t)$ is provided to the system through the vector $b_{j}$ at time $t$, and if $v_{j}(t)=0$, then the input $u_{j}(t)$ is not provided to the system. Thus Problem 1 can answer to a question of when and where exogenous control inputs should be provided. In other words, Problem 1 is considered as a node selection problem that extracts time-varying control nodes. To the best of our knowledge, the time-varying control node selection for continuous-time systems has not yet been proposed.

We next give an example of node selection problem based on the proposed method. We consider the network consisting of 5 nodes. Let $A$ in (2) be the adjacency matrix defined by

$$
A \triangleq\left[\begin{array}{lllll}
0 & 0 & 0 & 0 & 1 \\
0 & 0 & 1 & 1 & 0 \\
0 & 1 & 0 & 1 & 0 \\
0 & 0 & 1 & 0 & 0 \\
1 & 0 & 1 & 1 & 0
\end{array}\right]
$$




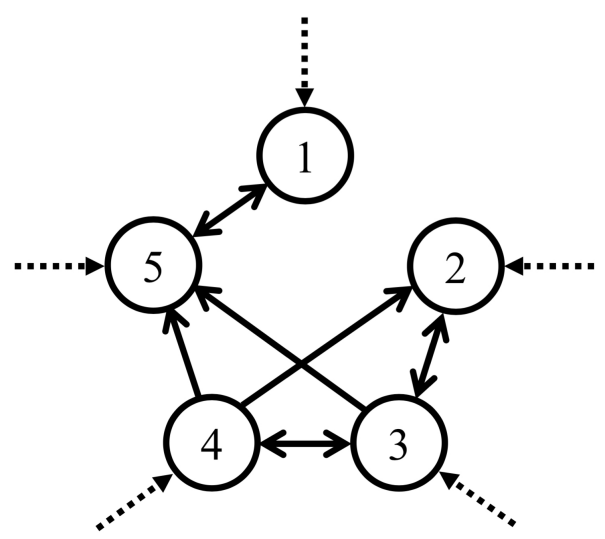

Fig. 1. Graph associated with $A$; Dashed lines show nodes that can be provided exogenous inputs.

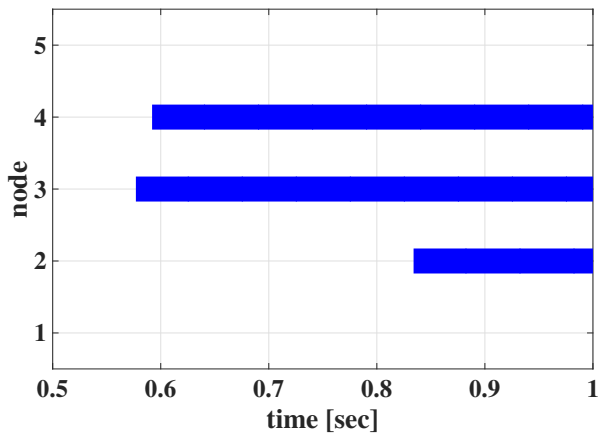

Fig. 2. Profile of control nodes

and take $B$ as $I_{5 \times 5}$, which is the identity matrix of dimension 5. Fig. 1 shows the graph according to the adjacency matrix A.

For this network, we simulated the proposed method with $T=1$ and $\alpha=1$. In this example, each node can be a control node since $B=I_{5 \times 5}$, but the constraint $\|v\|_{0} \leq \alpha=1$ imposes us to provide control inputs at most $1 \mathrm{sec}$. Note that this example satisfies the assumption of Theorem 2, and hence the optimal solution can be obtained by solving Problem 2, for which we can use, for example, CVX in MATLAB [24] after applying a time discretization.

Fig. 2 shows the profile of control nodes selected on the interval $[0, T]$. Note that we plot the profile only on $[0.5, T]$, since no node is activated on $[0,0.5]$ in this example. We can see that the set of control nodes is not constant on $[0, T]$. Indeed, the sets of control nodes on $[0.576,0.591)$, $[0.591,0.833)$, and $[0.833,1]$ are $\{3\},\{3,4\}$, and $\{2,3,4\}$, respectively. Note that our framework may select several nodes at the same time since $\|v\|_{0} \triangleq \sum_{j=1}^{m} \mu_{L}\left(\left\{t \in[0, T]: v_{j}(t) \neq\right.\right.$ $0\})$.

\section{CONCLusions}

In this paper, we have analyzed an optimal control problem that maximizes a controllability metric when a sparse control is applied. This analysis enables us to find an activation schedule of control inputs that can steer the system while saving energy. We have analytically shown the existence of the optimal solutions and proved that the solutions can be obtained via a convex optimization if each function (i.e. $f_{j}$ ) in front of decision variables is not constant. We have also illustrated the application of the optimization problem addressed in the paper to the control node selection problem. Our optimization can select time-varying control nodes for continuous-time systems.

\section{REFERENCES}

[1] G. Stadler, "Elliptic optimal control problems with $L^{1}$-control cost and applications for the placement of control devices," Computational Optimization and Applications, vol. 44, no. 2, pp. 159-181, 2009.

[2] R. Herzog, G. Stadler, and G. Wachsmuth, "Directional sparsity in optimal control of partial differential equations," SIAM Journal on Control and Optimization, vol. 50, no. 2, pp. 943-963, 2012.

[3] K. Kunisch, K. Pieper, and B. Vexler, "Measure valued directional sparsity for parabolic optimal control problems," SIAM Journal on Control and Optimization, vol. 52, no. 5, pp. 3078-3108, 2014.

[4] S. Boyd, M. T. Mueller, B. O'Donoghue, Y. Wang et al., "Performance bounds and suboptimal policies for multi-period investment," Foundations and Trends $($ in Optimization, vol. 1, no. 1, pp. 1-72, 2014.

[5] M. Nagahara, D. Quevedo, and J. Østergaard, "Sparse packetized predictive control for networked control over erasure channels," IEEE Trans. Autom. Control, vol. 59, no. 7, pp. 1899-1905, 2014.

[6] M. Nagahara, D. E. Quevedo, and D. Nešić, "Maximum hands-off control: a paradigm of control effort minimization," IEEE Trans. Autom. Control, vol. 61, no. 3, pp. 735-747, 2016.

[7] T. Ikeda and M. Nagahara, "Value function in maximum hands-off control for linear systems," Automatica, vol. 64, pp. 190-195, 2016.

[8] S. Sukumar and D. Chatterjee, "A jammer's perspective of reachability and LQ optimal control," Automatica, vol. 70, pp. 295-302, 2016.

[9] F. Pasqualetti, S. Zampieri, and F. Bullo, "Controllability metrics, limitations and algorithms for complex networks," IEEE Transactions on Control of Network Systems, vol. 1, no. 1, pp. 40-52, 2014.

[10] T. H. Summers, F. L. Cortesi, and J. Lygeros, "On submodularity and controllability in complex dynamical networks," IEEE Trans. Control Netw. Syst., vol. 3, no. 1, pp. 91-101, 2016.

[11] Y. Zhao, F. Pasqualetti, and J. Cortés, "Scheduling of control nodes for improved network controllability," in 55th IEEE Conference on Decision and Control (CDC), 2016, pp. 1859-1864.

[12] E. Nozari, F. Pasqualetti, and J. Cortés, "Time-invariant versus timevarying actuator scheduling in complex networks," in American Control Conference (ACC), 2017, pp. 4995-5000.

[13] M. R. Jovanovic and F. Lin, "Sparse quadratic regulator," in European Control Conference (ECC), 2013, pp. 1047-1052.

[14] T. Kailath, Linear systems. Prentice-Hall Englewood Cliffs, NJ, 1980, vol. 156.

[15] P. Müller and H. Weber, "Analysis and optimization of certain qualities of controllability and observability for linear dynamical systems," Automatica, vol. 8, no. 3, pp. 237-246, 1972.

[16] F. Clarke, Functional analysis, calculus of variations and optimal control. Springer Science \& Business Media, 2013, vol. 264.

[17] S. G. Krantz and H. R. Parks, A Primer of Real Analytic Functions. Springer Science \& Business Media, 2002.

[18] E. Hille and R. S. Phillips, Functional Analysis and Semi-groups. American Mathematical Soc., 1996, vol. 31

[19] A. Olshevsky, "Minimal controllability problems," IEEE Trans. Control Netw. Syst., vol. 1, pp. 249-258, 2014

[20] S. Assadi, S. Khanna, Y. Li, and V. M. Preciado, "Complexity of the minimum input selection problem for structural controllability," in IFACPapersOnLine, 2015, pp. 70-75.

[21] A. Clark, L. Bushnell, and R. Poovendran, "On leader selection for performance and controllability in multi-agent systems," in 51st IEEE Conference on Decision and Control (CDC), 2012, pp. 86-93.

[22] W. Yang, X. Wang, and H. Shi, "Optimal control nodes selection for consensus in multi-agent systems," IFAC Proceedings Volumes, vol. 47, no. 3, pp. 11697-11702, 2014.

[23] R. E. Kalman, "Mathematical description of linear dynamical systems," Journal of the Society for Industrial and Applied Mathematics, Series A: Control, vol. 1, no. 2, pp. 152-192, 1963.

[24] M. Grant and S. Boyd, "CVX: Matlab software for disciplined convex programming, version 2.1," http://cvxr.com/cvx, Mar. 2014 